\title{
Achieving seventh-order amplitude accuracy in leapfrog integrations
}

Paul Williams

University of Reading, Department of Meteorology, Reading, United Kingdom (p.d.williams@ reading.ac.uk)

The leapfrog time-stepping scheme is commonly used in general circulation models of weather and climate. The Robert-Asselin filter is used in conjunction with it, to damp the computational mode. Although the leapfrog scheme makes no amplitude errors when integrating linear oscillations, the Robert-Asselin filter introduces first-order amplitude errors. The RAW filter, which was recently proposed as an improvement, eliminates the first-order amplitude errors and yields third-order amplitude accuracy. This development has been shown to significantly increase the skill of medium-range weather forecasts. However, it has not previously been shown how to further improve the accuracy by eliminating the third- and higher-order amplitude errors.

This presentation will show that leapfrogging over a suitably weighted blend of the filtered and unfiltered tendencies eliminates the third-order amplitude errors and yields fifth-order amplitude accuracy. It will also show that the use of a more discriminating $(1,-4,6,-4,1)$ filter instead of a $(1,-2,1)$ filter eliminates the fifth-order amplitude errors and yields seventh-order amplitude accuracy. Other related schemes are obtained by varying the values of the filter parameters, and it is found that several combinations offer an appealing compromise of stability and accuracy. The proposed new schemes are shown to yield substantial forecast improvements in a medium-complexity atmospheric general circulation model. They appear to be attractive alternatives to the filtered leapfrog schemes currently used in many weather and climate models.

\section{References}

PD Williams (2013) Achieving seventh-order amplitude accuracy in leapfrog integrations. Monthly Weather Review 141(9), pp 3037-3051. doi:10.1175/MWR-D-12-00303.1

PD Williams (2011) The RAW filter: An improvement to the Robert-Asselin filter in semi-implicit integrations. Monthly Weather Review 139(6), pp 1996-2007. doi:10.1175/2010MWR3601.1

PD Williams (2009) A proposed modification to the Robert-Asselin time filter. Monthly Weather Review 137(8), pp 2538-2546. doi:10.1175/2009MWR2724.1 